\title{
SOP: antibody-associated autoimmune encephalitis
}

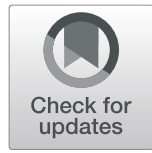

Rosa Rössling ${ }^{1,2}$ and Harald Prüss ${ }^{1,2^{*}}$

\begin{abstract}
Background: Antibody-mediated and paraneoplastic autoimmune encephalitides (AE) present with a broad spectrum of clinical symptoms. They often lead to progressing inflammatory changes of the central nervous system with subacute onset and can cause persistent brain damage. Thus, to promptly start the appropriate and AEspecific therapy, recognition of symptoms, initiation of relevant antibody diagnostics and confirmation of the clinical diagnosis are crucial, in particular as the diseases are relatively rare.

Aim: This standard operating procedure (SOP) should draw attention to the clinical presentation of $A E$, support the diagnostic approach to patients with suspected AE and guide through the necessary steps including therapeutic decisions, tumour screening and exclusion of differential diagnoses.

Method: Based on existing diagnostic algorithms, treatment recommendations and personal experiences, this SOP gives an overview of clinical presentation, diagnostic procedures and therapy in AE. Additional information is provided within an accompanying text and a table describing the most important autoantibodies and their characteristics.

Results: The initial steps of the AE flow chart are based on clinical symptoms and the patient's history. Assignment to paraneoplastic or antibody-mediated AE is sometimes clinically possible. Diagnostics should include MRI, EEG and CSF analysis with antibody panel diagnostic. Definite AE can be diagnosed if the underlying antibody is compatible with the clinical presentation. Classification of probable AE may be possible even with negative antineuronal autoantibodies if the clinical presentation and laboratory abnormalities are highly suggestive of AE. The confirmed AE diagnosis requires immediate initiation of immunotherapy.
\end{abstract}

Conclusion: The SOP facilitates the recognition of patients with AE and presents the necessary diagnostic and therapeutic steps.

Keywords: Autoimmune, Encephalitis, Limbic encephalitis, Antibody, Paraneoplastic, NMDAR

\section{Introduction}

Autoimmune encephalitis (AE) is an often rapidly progressive inflammatory neurological disease with subacute onset. The best characterized and most common form of $\mathrm{AE}$ is anti-NMDA receptor (NMDAR) encephalitis, defined by cerebrospinal fluid (CSF) IgG antibodies targeting the NMDA type glutamate receptor. Patients can present with altered mental status, reduced levels of consciousness, deficits in working/short-term memory that

\footnotetext{
*Correspondence: harald.pruess@charite.de

'Department of Neurology and Experimental Neurology, Charité -

Universitätsmedizin Berlin, CharitéCrossOver, R 4-334, Charitéplatz 1, 10117

Berlin, Germany

${ }^{2}$ German Center for Neurodegenerative Diseases (DZNE) Berlin, Berlin, Germany
}

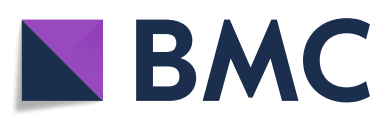

(๑) The Author(s). 2020 Open Access This article is distributed under the terms of the Creative Commons Attribution 4.0 International License (http://creativecommons.org/licenses/by/4.0/), which permits unrestricted use, distribution, and

reproduction in any medium, provided you give appropriate credit to the original author(s) and the source, provide a link to the Creative Commons license, and indicate if changes were made. The Creative Commons Public Domain Dedication waiver (http://creativecommons.org/publicdomain/zero/1.0/) applies to the data made available in this article, unless otherwise stated. $<6$ weeks), and may show psychosis or new epileptic seizures [1].

$\mathrm{AE}$ comprises antibody-mediated and paraneoplastic (i.e. usually cytotoxic T-cell-mediated) encephalitides, which form a heterogeneous group of autoimmune neuropsychiatric diseases with still broadening clinical phenotypes and numerous novel autoantibodies identified in recent years (Table 1). The incidence is estimated at 5-10 per 100.000 inhabitants per year [1] with often specific age and gender preferences for a given antibody.

Although much has been learned about AEs and their variable clinical phenotypes, diagnosis is often still delayed, partly related to incomplete awareness and false 
Table 1 Most important antibodies and clinical syndromes

\begin{tabular}{|c|c|c|c|c|}
\hline Antigen & Characteristics & $\begin{array}{l}\text { Preferred } \\
\text { detection }\end{array}$ & Age/Gender & Tumour \\
\hline \multicolumn{5}{|c|}{ Antibodies against neurotransmitter receptors [2] } \\
\hline NMDAR [3] & $\begin{array}{l}\text { Schizophreniform psychosis, perioral } \\
\text { dyskinesia, epileptic seizures, coma, } \\
\text { dystonia, hypoventilation; CMRI } \\
\text { frequently normal, often CSF } \\
\text { pleocytosis, EEG with slow waves, can } \\
\text { show extreme delta brush }\end{array}$ & CSF & $\begin{array}{l}\text { Most prevalent subtype of AE; } \\
\text { All ages, peak in childhood and } \\
\text { youth, } 75 \% \text { women }\end{array}$ & Ovarian teratoma \\
\hline GABAaR & $\begin{array}{l}\text { Epileptic seizures, schizophreniform } \\
\text { syndrome, refractory status epilepticus } \\
\text { and epilepsia partialis continua }\end{array}$ & Serum or CSF & Younger adults; $m>f(1.5: 1)$ & Hodgkin lymphoma \\
\hline GABAbR & LE with frequent epileptic seizures & CSF & Older adults $f=m$ & $50 \%$ lung cancer (SCLC) \\
\hline AMPAR & $\begin{array}{l}\text { LE, Epileptic seizures, memory deficits, } \\
\text { psychosis; CSF often normal }\end{array}$ & CSF & Older Adults $f>m(2.3: 1)$ & In $70 \%$ lung/ breast cancer \\
\hline mGluR5 & $\begin{array}{l}\text { LE, Ophelia syndrome (depression, } \\
\text { agitation, hallucination, memory deficits, } \\
\text { personality changes) }\end{array}$ & CSF & Young adults, $m>f,(1.5: 1)$ & Hodgkin lymphoma \\
\hline GlycinR & $\begin{array}{l}\text { PERM (progressive encephalomyelitis } \\
\text { with rigidity and myoclonus), SPS, } \\
\text { cognitive deficits }\end{array}$ & Serum or CSF & Older adults $f=m$ & Thymoma $(<10 \%)$ \\
\hline DPPX & $\begin{array}{l}\text { LE with tremor, myoclonus, hallucinations, } \\
\text { therapy refractory diarrhoea }\end{array}$ & CSF & Older adults $f<m(1: 2.3)$ & Not known \\
\hline \multicolumn{5}{|c|}{$\begin{array}{l}\text { Antibodies against ion channel subunits or cell adhesion } \\
\text { molecules }[4,5]\end{array}$} \\
\hline LGl1 & $\begin{array}{l}\text { Facio-brachial dystonic seizures, amnesia, } \\
\text { psychosis, LE, Medial temporal lobe } \\
\text { hyperintensities in MRI, hyponatremia }\end{array}$ & Serum & $\begin{array}{l}\text { Second most common type of AE; } \\
\text { Adults }>40 \text { years, } m>f(2: 1)\end{array}$ & Rare \\
\hline Caspr2 & $\begin{array}{l}\text { LE, neuromyotonia, Morvan syndrome, } \\
\text { can slowly progress over up to } 1 \text { year; } \\
\text { similar to LGI1, but no hyponatremia }\end{array}$ & Serum & Elderly $m>f(9: 1)$ & Thymoma possible \\
\hline IgLON5 & $\begin{array}{l}\text { REM- and non-REM sleep disorders, sleep } \\
\text { apnoea, stridor, dysarthria, dysphagia, } \\
\text { dysautonomia, movement disorders, } \\
\text { dementia }\end{array}$ & Serum & Older adults, $f=m$ & Not known \\
\hline \multicolumn{5}{|c|}{ Antibodies against glial structures } \\
\hline GFAP [6] & $\begin{array}{l}\text { Headache, subacute encephalopathy, } \\
\text { optic papillitis, myelitis, CS }\end{array}$ & Serum and CSF & $f=m$ & Possible \\
\hline \multicolumn{5}{|c|}{ Antibodies against Intracellular (onconeural) antigens $[7,8]$} \\
\hline $\mathrm{Hu}(\mathrm{ANNA}-1)$ & $\begin{array}{l}\text { Encephalomyelitis, brainstem encephalitis, } \\
\text { LE, Denny-Brown syndrome }\end{array}$ & Serum & $\begin{array}{l}\text { Large variability, depending on } \\
\text { tumour occurrence }\end{array}$ & $>90 \%, \mathrm{SCLC}$ \\
\hline Ri (ANNA-2) & OMS, CS, encephalomyelitis & Serum & & $>90 \%$, Ovary, breast cancer \\
\hline Yo (PCA-1) & CS & Serum & & $>90 \%$, Ovary cancer \\
\hline Ma2 & LE, CS, diencephalic/ hypothalamic involvement & Serum & & $>90 \%$, Testicular, lung cancer \\
\hline CV2 (CRMP5) & Encephalomyelitis, LE, CS & Serum & & $>90 \%$, SCLC, thymoma \\
\hline Amphiphysin & SPS & Serum & & $>90 \%$, Breast, SCLC \\
\hline GAD & SPS, LE, ataxia & Serum and CSF & Middle aged, $f>m(4: 1)$ & $\begin{array}{l}\text { Only rarely associated } \\
\text { with tumour }\end{array}$ \\
\hline
\end{tabular}

LE: limbic encephalitis, SPS: Stiff-person syndrome, OMS: Opsoclonus-myoclonus syndrome, CS: cerebellar syndrome, SCLC: small cell lung cancer, PCD: paraneoplastic cerebellar degeneration

assignment of the presented symptoms [9]. Early diagnosis, however, is crucial in order to promptly start the appropriate therapy which also depends on the type of AE. This SOP aims for facilitating the approach to patients with central nervous system symptoms suspicious of AE, for raising awareness to the clinical presentation of such patients, and for proposing the necessary diagnostic and therapeutic steps (Fig. 1).

Antibodies in antibody-mediated AE are mostly directed against neuronal surface antigens. By binding to 
excitatory transmitter receptors (NMDA, AMPA), inhibitory transmitter receptors (GABAb, GABAa, glycine), ion channel subunits and cell adhesion molecules (CASPR2, IgLON5) or soluble synaptic proteins (LGI1) they are directly pathogenic. Autoantibody formation can be triggered by viral or tumour exposition [10], but mechanisms are unknown in most cases. In contrast, antibodies in paraneoplastic neurological syndromes (PNS) bind to intracellular antigens and therefore cannot cause the disease directly, even though they serve as valuable biomarkers for an underlying tumour (such as $\mathrm{Hu}, \mathrm{Ri}$, Yo or Ma2 antibodies). Neuronal damage in these cases is rather caused by cytotoxic T-cells with oligoclonal $\mathrm{T}$-cell receptor expansion and autoreactivity against neuronal structures. Triggers for antibody production in PNS are presumably ectopically presented antigens on the tumour cells that are otherwise exclusively expressed in neurons. Parenchymal invasion of immune cells leads to early neuronal cell damage with consecutive cell death. Among the antibodies targeting intracellular antigens, GAD and amphiphysin antibodies are an exception as they seem to be pathogenically relevant despite their intracellular antigen location. Furthermore, GAD antibodies are only rarely associated with a tumour [11].

\section{Diagnosis}

Early diagnosis of antibody-mediated AE or PNS can be difficult because of the broad spectrum of disease manifestations. Clinical presentation is regularly not limited to a well-defined syndrome. However, there are some red flags that strongly indicate AE. These include common signs and symptoms, such as altered level of consciousness, dyskinesia, faciobrachial dystonic seizures, autonomic dysfunction, focal neurological signs, aphasia/dysarthria, hyponatremia, headache, catatonia or suspected malignant neuroleptic syndrome. In PNS every level of the nervous system can be affected. In this SOP, we focus on classical central nervous system presentations that include encephalomyelitis, limbic encephalitis, subacute cerebellar degeneration and opsoclonus-myoclonus syndrome. Additional autoimmune disorders in the patient's history (such as thyroid disease, diabetes, vitiligo or inflammatory bowel disease) suggest increased susceptibility to autoimmunity and should likewise prompt antibody testing even in the absence of abnormalities in MRI, EEG or CSF (Fig. 1).

"1 MRI is a central part of the standard work-up in $\mathrm{AE}$, even though imaging might only show non-specific changes in early stages of disease. For example, in NMDAR encephalitis, MRI is unremarkable in more than $50 \%$ of patients despite severe clinical symptoms. Similarly, at the beginning of clinical symptoms in paraneoplastic cerebellar degeneration (PCD), imaging might be normal and cerebellar atrophy is only visible later in the disease course. On the other hand, increased signal in T2-weighted/FLAIR imaging in the medial temporal lobes allows the diagnosis of 'definite limbic encephalitis' in the appropriate clinical context [1]. MRI in patients with GABAaR encephalitis is almost always abnormal with multifocal diffuse cortical and subcortical T2/ FLAIR hyperintensities. Severity of MR-morphological damage can correspond with prognosis. For instance, in LGI1 encephalitis bilateral hippocampal atrophy indicates poor outcome with persistent cognitive deficits. Furthermore, atrophy might progress during follow-up, even if prior imaging was unremarkable [12].

*2 EEG is also very helpful in PNS and antibodymediated AE. Although it is only rarely specific, EEG is altered in most patients and often shows general slowing or helps to detect subclinical seizures or a nonconvulsive status epilepticus. In patients with NMDAR encephalitis, an 'extreme delta brush' is a rare but specific finding that can lead to diagnosis [13].

"3 CSF analysis is always recommended in the workup of patients with suspected AE. Basic CSF parameters (white blood cells, protein) differ profoundly depending on the underlying type of AE. Inflammatory changes in the CSF are typically seen in patients with antibodies against NMDAR, GABAbR, AMPAR or DPPX, whereas antibodies targeting CASPR2, LGI1, GABAaR or glycine-R can be associated with normal CSF findings. CSF from IgLON5 antibody-positive patients typically shows increased protein. Oligoclonal bands occur mostly with antibodies associated with pleocytosis, an exception is GAD encephalitis, where oligoclonal bands without further CSF findings are typical [14]. For antibodies that are predominantly found in the CSF (such as NMDAR antibodies), titres are not only relevant for diagnostics, but also helpful during follow-up. In $14-20 \%$ of patients with anti-NMDAR encephalitis antibodies are present in CSF only. In contrast, serum levels of most antibodies do not correlate well with clinical course and prognosis. For some of them, antibody titres should reach certain levels to support the diagnosis. For example, serum Caspr2 antibodies $<1: 320$ or GAD antibodies $<2000 \mathrm{U} / \mathrm{ml}$ are mostly not sufficient to confirm the diagnosis of $\mathrm{AE}$.

"4 In every patient with a history suspicious for AE, a panel of autoantibodies should be analysed (Fig. 2), even if MRI, EEG and basic CSF parameters are normal. In suspected AE it is always recommended to test both serum and CSF to not overlook treatable conditions. Some clinicians test serum first and expand diagnosis to CSF if results were negative, however, this will normally delay diagnosis. Common panels of neuronal surface autoantibodies include detection of IgG against NMDAR, Caspr2, LGI1, GABAbR, AMPAR, followed by GABAaR, mGluR5, glycine-R and IgLON5.

*5 If clinical presentation matches a classical PNS or if a neoplasm is suspected, analysis of onconeural antibodies 


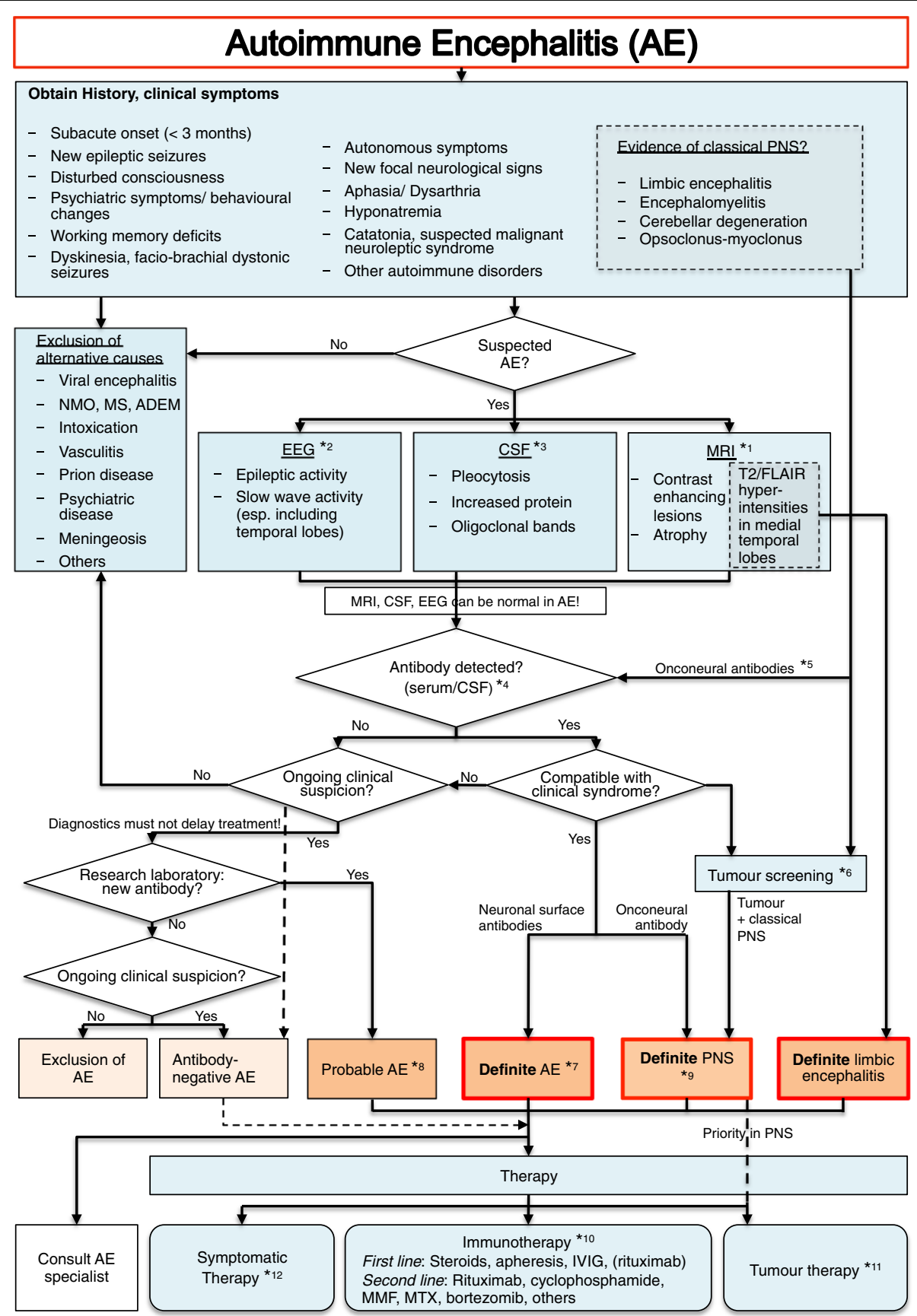

Fig. 1 Flow chart for the diagnosis of suspected autoimmune encephalitis. AE: Autoimmune encephalitis, PNS: paraneoplastic neurological syndrome, CSF: cerebrospinal fluid, FLAIR: fluid attenuated inversion recovery, MTX: methotrexate, MMF: mycophenolat mofetil, IVIG: intravenous immunoglobulin, NMO: neuromyelitis optica, MS: multiple sclerosis, ADEM: acute disseminated encephalomyelitis

is mandatory. Panel diagnostics including the following antibodies is recommended: $\mathrm{Hu}, \mathrm{Ri}, \mathrm{Yo}, \mathrm{Ma}, \mathrm{CV} 2$ and amphiphysin. Non-onconeural antibodies targeting neuronal surface antigens like GABAbR, AMPAR, mGluR5 and NMDAR (with the exception that tumours are typically teratomas) might also show high cancer association.

"6 In PNS, neurological symptoms often manifest weeks to months before recognition of the tumour. The likelihood of a tumour is $>95 \%$ when patients present with a classical PNS and a well-characterised onconeural antibody. The antibody itself may give a hint to the underlying tumour, e.g. Yo antibodies found in patients with PCD are characteristically associated with ovarian cancer. Standard imaging in tumour search includes body CT with contrast as well as MRI and ultrasound. If the findings are negative, a whole-body FDG-PET should 

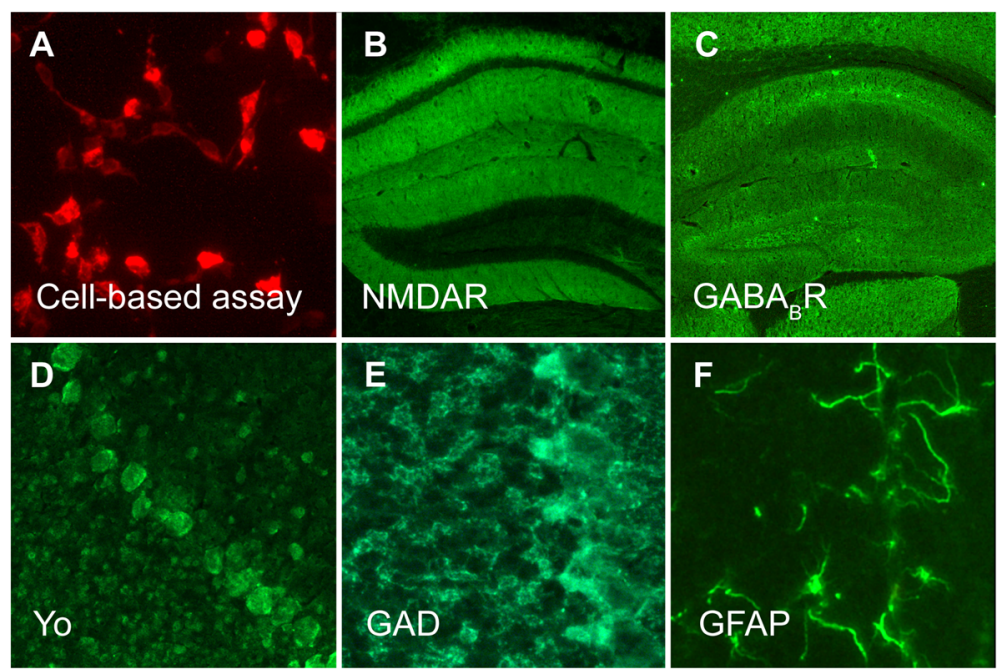

Fig. 2 Detection of anti-neuronal autoantibodies for the diagnosis of autoimmune encephalitis. a The current gold standard for established surface antibodies is the cell-based assay (CBA), in which diverse target antigens (in this example NMDAR) are recombinantly expressed on the surface of cultured cells. Binding of patient antibodies from CSF or serum samples can be visualized with fluorescent dyes. b The same CSF sample of a patient with NMDAR encephalitis shows strong binding on a mouse hippocampus section with the characteristic NMDAR distribution. c Autoantibodies to GABAbR also show strong binding to hippocampus tissue, but with a clearly distinguishable pattern. $\mathbf{d}$ Antibodies to onconeural antigens can be visualized by staining of line blots (not shown) or by their intracellular binding on brain sections, here Yo antibody-positive Purkinje neurons on a mouse cerebellum section. e GAD antibodies show a punctate pattern around cerebellar granule cells and Purkinje neurons. $\mathbf{f}$ Immunohistochemistry using brain sections also allows the detection of antibodies targeting glia cells, such as against GFAP. The methodology further permits detection of as yet undetermined anti-brain antibodies in research laboratories. D and $\mathrm{E}$ modified from "Prüss et al. 2017, Neurotransmitter"

be performed. At the beginning, tumours in PNS might be too small to be found, but as their detection is essential in providing the appropriate therapy, imaging should be repeated every 6 months for a minimum of three years [8].

"7 With confirmation of a specific antibody that is in line with the clinical syndrome, the diagnosis of 'definite AE' can be made. Examples include the presence of CSF NMDAR antibodies in a young woman with schizophreniform psychosis, epileptic seizures and hypoventilation, the presence of GABAaR antibodies in a middle-aged man with new onset epilepsy progressing to status epilepticus, or the detection of LGI1 antibodies in an older adult with new-onset amnesia, hyponatremia and marked behavioural changes. Immediate immunotherapy is required in almost all cases with 'definite $\mathrm{AE}$ '.

"8 We propose the diagnosis of 'probable AE' also in clinical constellations that are supported by the finding of a novel neuronal surface autoantibody in a research laboratory, using immunohistochemistry on brain sections (Fig. 2) or staining of live neurons. We generally recommend the CSF (+/- serum) analysis of unclear cases on a research basis if commercial antibody assays show negative results. Importantly, treatment should be initiated similar to definite $\mathrm{AE}$ and must not be delayed by an extended antibody search. The diagnosis of 'antibody-negative $\mathrm{AE}$ ' can be made in the absence of an anti-neuronal antibody if findings from clinical presentation, MRI or CSF strongly suggest an autoimmune aetiology and after exclusion of differential diagnoses [1].

"9 Diagnosis of 'definite PNS' according to Graus et al. [7] can be made in clinical constellations of $\mathrm{AE}$ where [1] the classical PNS and cancer develop within five years of the PNS diagnosis or [9] the neurological syndrome is associated with well-characterised onconeural antibodies (anti-Hu, Yo, CV2, Ri, Ma2 or amphiphysin), even in the absence of cancer.

\section{Therapy}

It is the nature of $\mathrm{AE}$ that brain dysfunction frequently leads to psychiatric symptoms with patient's rejection of consent for immediate diagnostics and treatment, comparable to infectious encephalitis. This is particularly important for clinical practice as delayed treatment of $\mathrm{AE}$ will result in irreversible damage and as the relative novelty of $\mathrm{AE}$ often leads to uncertainty about the patient's expressed will in clinical practice. We recently suggested an approach for AE patients with the lack of ability to give consent, but treatment is medically reasonable or even demanded [15].

"10 Therapy depends on the clinical syndrome and the underlying antibody. In patients with $\mathrm{AE}$ caused by antibodies against neuronal surface antigens, immunotherapy is usually more successful than in patients with 
antibodies targeting intracellular proteins. Treatment studies in patients with $\mathrm{AE}$ are sparse and focus on the most common forms of AE, such as NMDAR encephalitis. Although early therapy is critical, marked recovery can be seen in some patients with antibody-mediated $\mathrm{AE}$ in whom therapy is only started months after disease onset. First-line therapy in antibody-mediated AE comprises high-dose intravenous methylprednisolone (1000 $\mathrm{mg} / \mathrm{d}$ i.v. for 5 days), therapeutic apheresis (at least 5 times every other day, in cases with predominant CSF antibodies usually 7-10 treatments needed) or intravenous immunoglobulins $(2 \mathrm{~g} / \mathrm{kg}$ body weight over $3-5$ days). If no treatment effect is seen after two weeks, second-line therapy should be started with no delay. The anti-CD20 antibody rituximab is frequently used (1000 $\mathrm{mg}$, with the first two administrations at day 1 and day 15 followed by 6 months intervals). Due to its good tolerance and efficacy, many centres use rituximab as firstline therapy in $\mathrm{AE}$ patients with surface autoantibodies. Steroids might be sufficient in patients with LGI1 antibodies, but clearly not in patients with NMDAR encephalitis who should receive rituximab also to prevent relapses. If high antibody titres persist parallel to clinical symptoms, repeated apheresis should be considered. Antibody-mediated AE can be monophasic, i.e. maintenance treatments can often be stopped after 1-3 years. Comparative studies of the respective therapeutic option are still lacking. In any case, early initiation of immunotherapy is crucial not only regarding the acute phase of the disease, but also for long-term outcome. As shown in patients with NMDAR encephalitis, long-term outcome might be impaired by persistent cognitive deficits [16].

Cyclophosphamide is another option for second-line therapy and might be combined with rituximab. Many other treatments have been used with variable success, including mycophenolat mofetil, methotrexate or azathioprine. Promising new data suggest that the proteasome inhibitor bortezomib might be a valuable option in patients with surface antibody-mediated AE [17]. Given the ongoing expansion of immunotherapies in these indications (e.g. daratumumab, tocilizumab or autologous stem cell transplantation) and the right of all patients with rare $\mathrm{AE}$ to potentially receive such treatments, consultation of a specialised $A E$ centre is generally recommended after confirmation of diagnosis.

In paraneoplastic $\mathrm{AE}$ with antibodies targeting intracellular proteins, rituximab, intravenous immunoglobulins and therapeutic apheresis often have only little effect as the antibodies are not directly pathogenic, but neuronal damage is caused by cytotoxic T-cells. Furthermore, therapy in PNS is often delayed and substantial irreversible neuronal cell damage has already occurred at the time of presentation. If cell damage is visibly progressing in brain imaging after 3 to 6 months in spite of advanced immunotherapy, discontinuation of therapy is recommended in these patients.

"11 Evidence of a tumour requires, if possible, prompt and complete removal to withdraw the auto-antigen that is ectopically produced on tumour cells and likely triggers the production of autoantibodies. However, neuronal damage will often progress, especially in PNS [7]. Please see Table 1 for common associations of an antibody with a specific tumour.

"12 Symptomatic therapy depends on the form of AE. Antiepileptic therapy is frequently required as $\mathrm{AE}$ commonly leads to epileptic seizures. Antiepileptic drugs should be tapered after the encephalitic phase given that in surface antibody-mediated AE seizures are mainly acute-symptomatic. Psychotic symptoms often require transient treatment with antipsychotic drugs, which might also be tapered after the initial disease phase. With status epilepticus, autonomous symptoms or major behavioural abnormalities, patients regularly require intensive care unit treatment including sedation and mechanical ventilation. Physiotherapy and speech therapy can further help to improve the outcome.

\section{Conclusion}

In patients with suspected antibody-associated $\mathrm{AE}$, it is essential to analyse the patient's history for the abovementioned red flags. Standard diagnostic work-up includes EEG, MRI, CSF analysis and testing for anti-neuronal autoantibodies. 'Definite AE' or 'definite PNS' can be diagnosed when a detected antibody is compatible with the clinical syndrome. Treatment should be initiated as soon as possible and must not await pending antibody analysis. Consultation of an $\mathrm{AE}$ specialist is generally recommended after confirmation of the diagnosis of AE.

\section{Acknowledgements \\ Not applicable.}

Authors' contributions

R.R. and H.P. contributed to the conception and design of the work, analysed data, drafted the text and prepared the figures.

\section{Funding}

This work was supported by grants from the German Research Foundation (DFG) to H.P. (PR1274/2-1 and PR1274/3-1).

\section{Availability of data and materials \\ Available to readers on request. \\ The authors declare that they have no competing interests.}

Ethics approval and consent to participate

Not applicable.

Consent for publication

Not applicable.

Competing interests

The authors declare that they have no competing interests. 
Received: 6 October 2019 Accepted: 28 November 2019

Published online: 15 January 2020

\section{References}

1. Graus, F., Titulaer, M. J., Balu, R., et al. (2016). A clinical approach to diagnosis of autoimmune encephalitis. Lancet Neurol, 15, 391-404.

2. Dalmau, J., \& Graus, F. (2018). Antibody-mediated encephalitis. New England $J$ Med, 378, 840-851.

3. Dalmau, J., Armangue, T., Planaguma, J., et al. (2019). An update on antiNMDA receptor encephalitis for neurologists and psychiatrists: Mechanisms and models. Lancet Neurol.

4. Gaig, C., Graus, F., Compta, Y., et al. (2017). Clinical manifestations of the anti-IgLON5 disease. Neurol, 88, 1736-1743.

5. Irani, S. R., Alexander, S., Waters, P., et al. (2010). Antibodies to Kv1 potassium channel-complex proteins leucine-rich, glioma inactivated 1 protein and contactin-associated protein-2 in limbic encephalitis, Morvan's syndrome and acquired neuromyotonia. Brain, 133, 2734-2748.

6. Fang, B., McKeon, A., Hinson, S. R., et al. (2016). Autoimmune glial Fibrillary acidic protein Astrocytopathy: A novel Meningoencephalomyelitis. JAMA Neurol, 73, 1297-1307.

7. Graus, F., Delattre, J. Y., Antoine, J. C., et al. (2004). Recommended diagnostic criteria for paraneoplastic neurological syndromes. J Neurol, Neurosurg, Psychiatry, 75, 1135-1140.

8. Gultekin, S. H., Rosenfeld, M. R., Voltz, R., Eichen, J., Posner, J. B., \& Dalmau, J. (2000). Paraneoplastic limbic encephalitis: Neurological symptoms, immunological findings and tumour association in 50 patients. Brain, $123(\mathrm{Pt}$ 7), $1481-1494$

9. Herken, J., \& Prüss, H. (2017). Red flags: Clinical signs for identifying autoimmune encephalitis in psychiatric patients. Frontiers in Psychiatry, 8, 25.

10. Prüss, H., Finke, C., Holtje, M., et al. (2012). N-methyl-D-aspartate receptor antibodies in herpes simplex encephalitis. Annals Neurol, 72, 902-911.

11. Saiz, A., Blanco, Y., Sabater, L., et al. (2008). Spectrum of neurological syndromes associated with glutamic acid decarboxylase antibodies: Diagnostic clues for this association. Brain, 131, 2553-2563.

12. Heine, J., Prüss, H., Bartsch, T., Ploner, C. J., Paul, F., \& Finke, C. (2015), Imaging of autoimmune encephalitis--relevance for clinical practice and hippocampal function. Neurosci, 309, 68-83.

13. Schmitt, S. E., Pargeon, K., Frechette, E. S., Hirsch, L. J., Dalmau, J., \& Friedman, D. (2012). Extreme delta brush: A unique EEG pattern in adults with anti-NMDA receptor encephalitis. Neurol, 79, 1094-1100.

14. Blinder, T., \& Lewerenz, J. (2019). Cerebrospinal fluid findings in patients with autoimmune encephalitis-a systematic analysis. Frontiers in Neurol, 10, 804

15. Prüss, H., Kohler, S., \& Muller, S. (2019). Autoimmune encephalitis-Diagnostic and therapeutic decision tree from a psychiatric, neurological and ethicolegal point of view : Approach in cases of lack of ability to give consent and permissibility of compulsory treatment. Nervenarzt.

16. Finke, C., Kopp, U. A., Prüss, H., Dalmau, J., Wandinger, K. P., \& Ploner, C. J. (2012). Cognitive deficits following anti-NMDA receptor encephalitis. J Neurol, Neurosur, Psychiatry, 83, 195-198.

17. Scheibe, F., Prüss, H., Mengel, A. M., et al. (2017). Bortezomib for treatment of therapy-refractory anti-NMDA receptor encephalitis. Neurol, 88, 366-370.

\section{Publisher's Note}

Springer Nature remains neutral with regard to jurisdictional claims in published maps and institutional affiliations. 